\title{
DESTERPENAÇÃO POR EXTRAÇÃO LÍQUIDO-LÍQUIDO DE MISTURA MODELO DE ÓLEO DE BERGAMOTA EM COLUNA PRDC
}

\author{
C. C. KOSHIMA, D. GONÇALVES, K. T. NAKAMOTO e C. E. C RODRIGUES
}

\author{
Universidade de São Paulo, Faculdade de Zootecnia e Engenharia de Alimentos, Departamento de \\ Engenharia de Alimentos \\ E-mail para contato: Cristina.chiyoda@usp.br
}

\begin{abstract}
RESUMO - Hidrocarbonetos terpênicos possuem baixo poder aromatizante e sofrem maior degradação. Para aumentar a qualidade dos óleos essenciais, a redução destes compostos pode ser realizada por extração líquida utilizando etanol hidratado como solvente. A mistura modelo contendo limoneno, acetato de linalila e linalol foi desterpenada a $25 \pm 0,1{ }^{\circ} \mathrm{C}$ e $100 \mathrm{kPa}$ em coluna PRDC (Perforated Rotating Disc Contactor) utilizando etanol hidratado (30 e $40 \%$ de água, em massa), duas razões solvente/óleo (2 e 3) e três velocidades de rotação dos discos perfurados (80, 100 e 150 $\mathrm{rpm})$. Maiores valores de coeficiente global volumétrico de transferência de massa $(\mathrm{kg}$ linalol $/ \mathrm{m}^{3} \mathrm{~s} \mathrm{~kg}$ linalol $/ \mathrm{kg}$ fase tepênica) estão associados às maiores razões $(0,10$ para solvente $40 \%$ e 0,17 para solvente $30 \%)$, rotação $(0,12$ para solvente $40 \%$ e 0,18 para solvente $30 \%$ ) e menor grau de hidratação do etanol. Esses cálculos permitem avaliar a eficiência da extração, sendo importantes para planejar e otimizar o processo de separação.
\end{abstract}

\section{INTRODUÇÃO}

Óleos essenciais, de maneira geral, apresentam-se como misturas oleosas constituídas por muitos compostos químicos que variam desde hidrocarbonetos terpênicos, alcoóis simples e terpênicos, aldeídos, cetonas, fenóis, ésteres, éteres, óxidos, peróxidos, furanos, ácidos orgânicos, lactonas, cumarinas e, também, compostos com enxofre (Serafini et al., 2002).

A fração volátil dos óleos proveniente de frutas cítricas é composta por hidrocarbonetos terpênicos que, normalmente, apresentam teor maior que 90 \% (Haypek et al., 2000) e seus derivados oxigenados, que constituem geralmente cerca de $1 \%$ da mistura e são os principais responsáveis pelo aroma característico do óleo (Arce et al., 2003, 2005). Segundo Lago et al. (2012), o teor dos compostos oxigenados tornou-se um indicador da qualidade do óleo essencial, bem como um parâmetro para o estabelecimento do preço. Por outro lado, os hidrocarbonetos terpênicos tendem a se decompor na presença de calor e oxigênio, gerando odores desagradáveis os quais podem contribuir para a perda de qualidade do óleo (Gironi e Maschietti, 2012).

Quando comparado com outros óleos cítricos, o óleo essencial de bergamota contem menor teor 


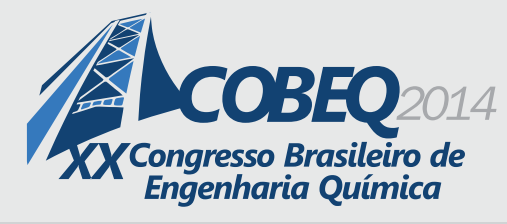

de hidrocarbonetos terpênicos e maiores quantidade de compostos oxigenados. Entretanto, para a sua utilização industrial como agente aromático, é interessante que este óleo seja submetido à desterpenação, visando à obtenção de uma fração enriquecida em compostos oxigenados. Esta fração é a qual é mais estável e mais solúvel em água e mantém, ao mesmo tempo, o aroma característico e fragrância do óleo bruto (Gironi e Maschietti, 2008).

Dentre os muitos procedimentos propostos para a desterpenação de óleos essenciais, em termos de aquisições de duas frações, uma delas rica em hidrocarbonetos terpênicos e a outra em compostos oxigenados, destaca-se a extração com solventes, ou extração líquido-líquido, pois pode ser conduzida a temperatura ambiente e pressão atmosférica (Arce et al., 2006), sendo as condições brandas de operação essenciais para a manutenção das propriedades sensoriais dos óleos (Haypek et al., 2000).

A extração líquido-líquido está fundamentada nas diferentes miscibilidades apresentadas entre os compostos presentes em uma mistura líquida (alimentação) em um dado solvente, também na fase líquida. Assim, a separação dos constituintes da alimentação se deve ao contato íntimo desta com um solvente que possua a capacidade de extrair preferencialmente um ou mais componentes desejados, os solutos. Os dois líquidos em contato, alimentação e solvente, precisam ser imiscíveis ou parcialmente miscíveis para que extração dos constituintes e, posteriormente, a separação física das duas fases formadas, extrato e rafinado, ocorra (Treybal, 1980).

O uso de soluções alcoólicas contendo etanol e água como solvente para a extração líquida é de grande interesse, pois a etapa de dessolventização, dependendo da aplicação, pode não ser necessária. De fato, extratos alcoólicos de óleos essenciais são altamente solicitados pelas indústrias devido à alta solubilidade apresentada em soluções aquosas, podendo, assim, ser facilmente adicionados a bebidas e perfumes. Além disso, estes extratos apresentam grande poder aromático e maior estabilidade devido ao fato das reações de oxidação serem reduzidas na presença de etanol (Li e Tamura, 2008).

Com base nas características físico-químicas do sistema a ser trabalhado, diferentes tipos de equipamentos podem ser empregados na extração com solventes, a saber: misturadores-decantadores, extratores centrífugos e colunas de extração (Aguilar e Cortina, 2008). As colunas de extração apresentam a vantagem das fases se separarem facilmente e rapidamente pela força da gravidade, sem necessidade de uma centrifuga, sendo o processo simples e fácil de operar em modo contínuo (Porto et al., 2010)

Dentre as diversas configurações de colunas extratoras, destaca-se a coluna de discos rotativos perfurados (Perforated rotating disc contactor, PRDC), pois apresenta vantagens em relação à simplicidade de construção, consumo de energia relativamente baixo, aumento da taxa e eficiência de transferência de massa que as perfurações proporcionam e alta eficiência proporcionada pelo movimento circular dos discos rotativos, os quais aumentam a turbulência do sistema (Porto et al., 2010).

Os trabalhos existentes na literatura sobre desterpenação de óleos essenciais por extração líquido - líquido são limitados e, em sua maioria, se concentram na determinação experimental e modelagem de dados de equilíbrio líquido-líquido de sistemas modelo compostos por representante 
da classe de terpenos e oxigenados. Estudos que reportem informações sobre o comportamento do sistema e a viabilidade do processo em equipamentos de extração são raros ou mesmo inexistentes. Sendo assim, o objetivo deste trabalho foi avaliar a transferência de massa em uma coluna de discos rotativos perfurados (PRDC) no processo de desterpenação por extração líquido-líquido de óleo essencial de bergamota modelo composto por limoneno, acetato de linalila e linalol.

\section{MÉTODOS}

\subsection{Operação da coluna de discos rotativos perfurados (PRDC)}

Para o estudo do processo de extração líquido-líquido em PRDC foi utilizada um equipamento (Figura 1) composto por três partes: região de extração $(\mathrm{H}=16 \mathrm{~cm}$, diâmetro interno, $\mathrm{DI}=2,8 \mathrm{~cm})$; região de separação superior $(\mathrm{H}=14 \mathrm{~cm}$, diâmetro externo, $\mathrm{DE}=5 \mathrm{~cm}$, e interno $\mathrm{DI}=2,8 \mathrm{~cm})$; região de separação inferior $(\mathrm{H}=10,5 \mathrm{~cm} ; \mathrm{DE}=5,0 \mathrm{~cm} ; \mathrm{DI}=2,8 \mathrm{~cm}$ e um cilindro $1,60 \mathrm{~cm}$ de diâmetro para a entrada de alimentação). A coluna é encamisada permitindo o controle de temperatura. Apresenta 10 discos perfurados na região de extração, $\operatorname{com} 1,31 \mathrm{~cm}$ de distância entre os discos. Cada disco apresenta 2,65 $\mathrm{cm}$ de DE, $0,35 \mathrm{~cm}$ de DI, espessura de $0,15 \mathrm{~cm}$ e 33 perfurações de $0,25 \mathrm{~cm}$ de diâmetro $\left(1,62 \mathrm{~cm}^{2}\right.$ de área livre de escoamento).

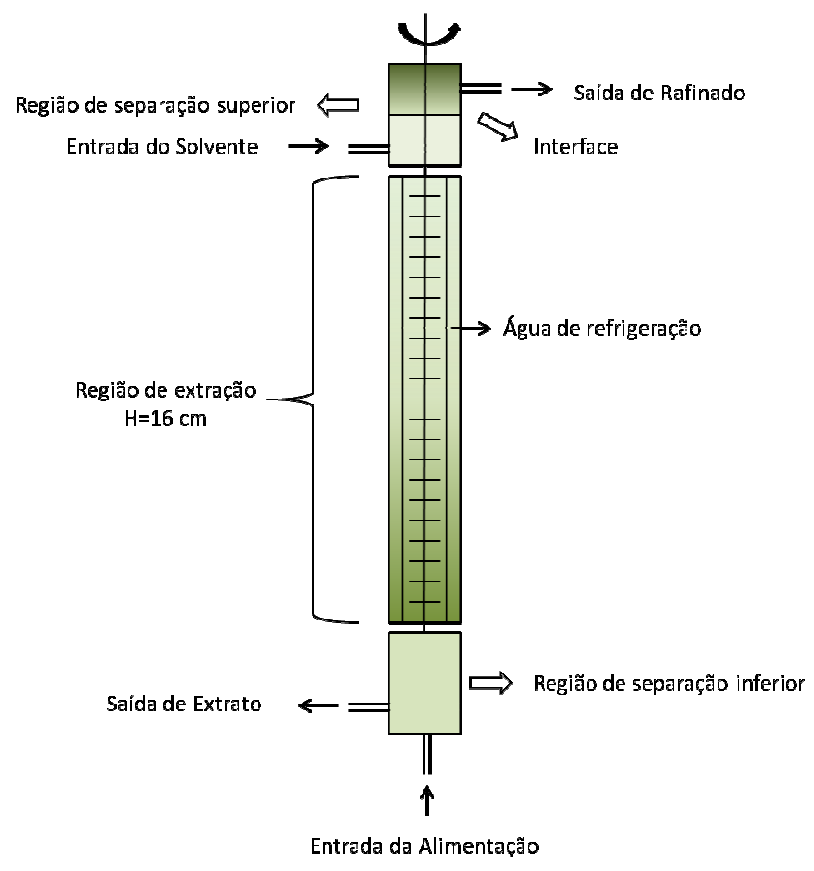

Adaptado de Rodrigues, 2004

Figura 1 - Esquema da coluna de discos rotativos perfurados.

A fase contínua, solvente alcoólico, composto por etanol absoluto (Merck, $\geq 99,8 \%$, CAS 6417-5) e água deionizada (Millipore, modelo Direct-Q3, França), foi introduzida na região superior da coluna e seu fluxo foi mantido constante em valor pré-estabelecido. O agitador (IKA, modelo 


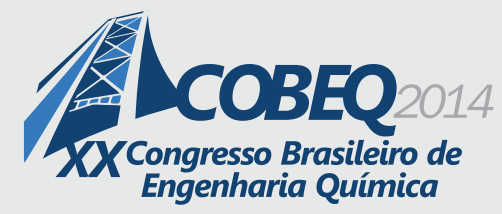

Eurostar power control visc P7, EUA) foi ligado e a velocidade de rotação pré-estipulada foi selecionada e medida através do tacômetro digital (Icel, modelo TC-5010, Brasil). Em seguida, o equipamento foi alimentado com a fase dispersa, óleo essencial de bergamota modelo, composto por $5 \%$ de linalol (Sigma-Aldrich, 97\%, CAS 78-70-6), 45,24\% de acetato de linalila (Sigma-Aldrich, 97\%, CAS 115-95-7) e 49,76\% de limoneno (Sigma-Aldrich, 97-98 \%, CAS 5989-27-5) no fundo da coluna e seu fluxo foi mantido constante em valor pré-estabelecido durante todo experimento. Ambas as entradas foram introduzidas na coluna com o auxílio de bombas peristálticas digitais (Cole Parmer, modelo 77200-60, EUA).

O início da retirada das amostras se deu após o estabelecimento da interface na região de separação superior. Cinco amostras das correntes das fases extrato e rafinado foram retiradas dos compartimentos inferior e superior da coluna, respectivamente; pesadas para a determinação das vazões de saída e submetidas às análises de CG-DIC e titulação Karl Fischer para a obtenção das composições das fases, conforme sugerido por Chiyoda et al. (2011).

Foram realizados 6 experimentos em duplicata conduzidos a pressão atmosférica local (100 $\mathrm{kPa}$ ) e utilizando a temperatura da água da camisa igual a $25,0 \pm 0,1{ }^{\circ} \mathrm{C}$. As demais condições experimentais usadas em cada um dos experimentos podem ser verificadas na Tabela 1.

Tabela 1 - Condições experimentais utilizadas nos experimentos de desterpenação de óleo modelo de bergamota

\begin{tabular}{cccc}
\hline Experimento & $\begin{array}{c}\text { Teor mássico de água no } \\
\text { solvente etanólico }(\%)\end{array}$ & $\begin{array}{c}\text { Velocidade de rotação } \\
\text { dos discos }(\mathrm{rpm})\end{array}$ & $\begin{array}{c}\text { Razão solvente/ } \\
\text { alimentação }\end{array}$ \\
\hline 1 & 40 & 100 & 2 \\
2 & 40 & 100 & 3 \\
3 & 40 & 150 & 3 \\
4 & 30 & 80 & 2 \\
5 & 30 & 80 & 3 \\
6 & 30 & 100 & 2 \\
\hline
\end{tabular}

\section{RESULTADOS E DISCUSSÃO}

Com a finalidade de avaliar a transferência dos compostos presentes no sistema, índices de extração (IE) dos compostos terpênicos para o extrato, ou fase solvente, foram calculados de acordo com a Equação 1, onde $\mathrm{F}$ e $\mathrm{E}$, são as vazões mássicas da correntes alimentação e extrato (fase solvente), $\mathrm{w}_{\mathrm{i}}$ é a fração mássica do componente $\mathrm{i}$.

$$
I E(\%)=\frac{E w_{i}^{E}}{F w_{i}^{F}} \times 100
$$

O impacto das variáveis de processo razão solvente/ alimentação, velocidade de rotação dos discos perfurados e teor de hidratação do solvente sobre os índices de extração (IE) podem ser visualizados na Figura 2. 

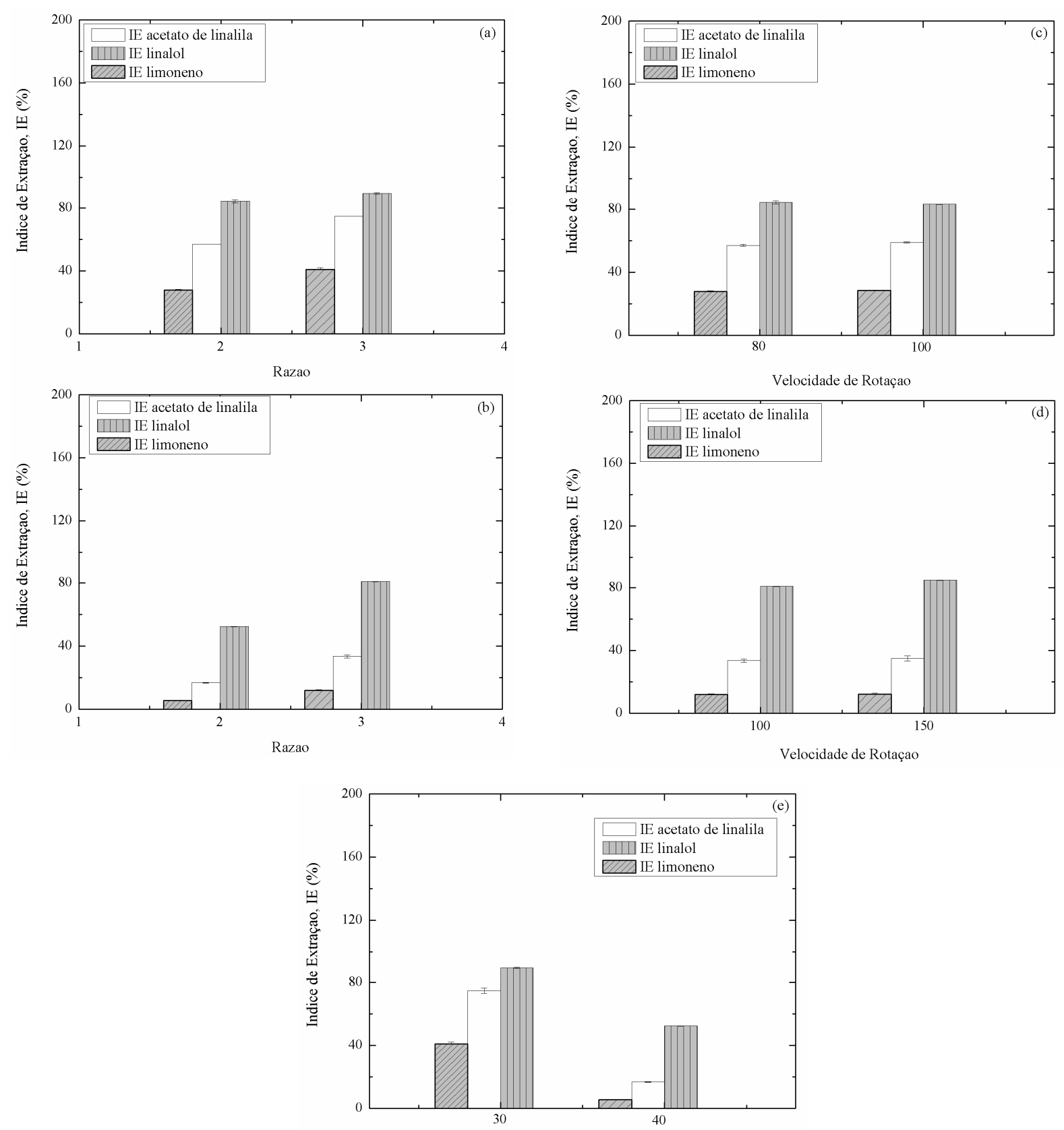

Teor Massico de Agua no Solvente (\%)

Figura 2 - Comparação dos índices de extração dos compostos terpênicos para a fase solvente ou extrato. (a) Efeito da razão para solvente $30 \%$ e $80 \mathrm{rpm}$; (b) Efeito da razão para solvente $40 \%$ e 100 rpm; (c) Efeito da velocidade de rotação dos discos para solvente $30 \%$ e razão 2; (d) Efeito da velocidade de rotação dos discos para solvente 40 \% e razão 3; (e) Efeito da hidratação do solvente para 100 rpm e razão 2 . 


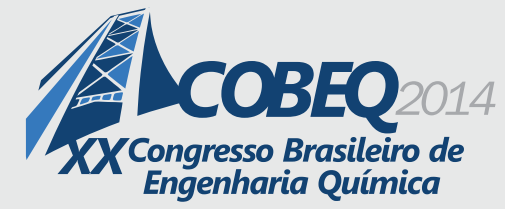

É sendo possível inferir, com base na Figura 2, que o linalol migra mais para a fase solvente ou extrato seguido pelo acetato de linalila e limoneno. De fato, no estudo do equilíbrio líquido-líquido dos sistemas de óleo de bergamota modelo (Chiyoda et al., 2011), foi observado, para os compostos do óleo, um maior valor de $\mathrm{k}$ para linalol, seguido do acetato de linalila e limoneno.

$\mathrm{Na}$ Figura 2, independentemente do solvente estudado, maiores índices de extração estão associados ao maior valor de razão. Por outro lado, o efeito da velocidade de rotação dos discos parece não apresentar impacto significativo sobre os índices calculados. Com relação aos diferentes níveis de hidratação do etanol, nota-se que o aumento do teor de água no solvente é responsável pela diminuição dos índices de extração dos compostos terpênicos para a fase solvente.

A observação sobre a diminuição da extração dos compostos do óleo com a maior hidratação do solvente está de acordo com o estudo do equilíbrio de fase dos sistemas de óleo de bergamota modelo (Chiyoda et al., 2011), uma vez que a água diminui a solubilidade das fases o que leva a uma menor partição dos componentes.

A transferência de massa também foi avaliada por meio dos coeficientes volumétricos globais de transferência de massa (TM), estimados via Equação 2. Deve-se considerar que os efeitos de dispersão axial e da transferência de outros componentes não foram levados em conta nos cálculos, desta forma os valores calculados podem ser considerados como coeficientes globais aparentes. Apesar destas aproximações, os resultados obtidos podem possibilitar o projeto de extratores líquidolíquido para desterpenação de óleos essenciais.

$$
R^{\prime}\left(w_{F, 2}^{\prime}-w_{R, 2}^{\prime}\right)=K_{R} a V \Delta w_{R, 2 M}^{\prime}
$$

onde: $R$ ' é a vazão mássica da fase rafinado em base livre de linalol em $\mathrm{kg} / \mathrm{s}, w_{F, 2}^{\prime}$ e $w_{R, 2}^{\prime}$ são as concentrações de linalol nas correntes de alimentação e rafinado, respectivamente, $K_{R}$ é o coeficiente de TM global em $\mathrm{kg}$ linalol $/\left(\mathrm{m}^{2} . \mathrm{s} .(\mathrm{kg}\right.$ linalol $/ \mathrm{kg}$ fase terpênica), $a$ é a área de TM por unidade de volume da zona de extração em $\mathrm{m}^{2} / \mathrm{m}^{3}, V$ é o volume da zona de extração $\mathrm{em} \mathrm{m}^{3} \mathrm{e} \Delta w_{R, 2 M}$ é a média logarítmica das diferenças de concentração nos extremos da coluna baseada nas unidades de concentração da fase terpênica. $\Delta \mathrm{w}_{\mathrm{R}, 2 \mathrm{M}}$ foi calculado levando-se em consideração as concentrações de equilíbrio dadas pelas Equações 3 e 4 (Treybal, 1980).

As curvas de equilíbrio líquido-líquido, a $25,0 \pm 0,1{ }^{\circ} \mathrm{C}$, de sistemas contendo limoneno + acetato de linalila + linalol + etanol + água foram obtidas por Chiyoda et al. (2011). As curvas de equilíbrio, para os solventes com 30,85 (Equação 3) e 42,15 \% (Equação 4) de água, em massa, são parábolas com um coeficiente de correlação de 0,9999 e 0,9985 , respectivamente. A faixa de concentração de linalol estudada foi de $0-10 \%$ e $0-15 \%$ para o etanol com 30,85 e $42,15 \%$ de água, respectivamente.

$$
\begin{aligned}
& w_{E, 2}^{\prime}=-0,8599 w_{R, 2}^{\prime}{ }^{2}+0,4852 w_{R, 2}^{\prime} \\
& w_{E, 2}^{\prime}=-0,4232 w_{R, 2}^{\prime}+0,2002 w_{R, 2}^{\prime}
\end{aligned}
$$


As frações mássicas utilizadas nas Equações 2 a 4 estão em base livre de linalol. Tais composições são calculadas através da Equação 5.

$$
w_{2}^{\prime}=\frac{w_{2}}{1-w_{2}}
$$

Os coeficientes globais volumétricos de transferência de massa $\left(K_{R} a\right)$ são mostrados na Tabela 2.

Tabela 2 - Valores para os coeficientes globais volumétricos de transferência de massa $\left(K_{R} a\right)$

\begin{tabular}{ccccc}
\hline Exp. & $\begin{array}{c}\text { Teor de água no } \\
\text { solvente }(\%)\end{array}$ & $\begin{array}{c}\text { Velocidade rotação } \\
\text { dos discos }(\mathrm{rpm})\end{array}$ & $\begin{array}{c}\text { Razão solvente/ } \\
\text { alimentação }\end{array}$ & $\begin{array}{c}K_{R} a\left[\mathrm{~kg} \text { linalol/ } \mathrm{m}^{3} \mathrm{~s}(\mathrm{~kg}\right. \\
\text { linalol } / \mathrm{kg} \text { fase tepênica })\end{array}$ \\
\hline 1 & 40 & 100 & 2 & $0,0726 \pm 0,0018$ \\
2 & 40 & 100 & 3 & $0,0979 \pm 0,0028$ \\
3 & 40 & 150 & 3 & $0,1208 \pm 0,0085$ \\
4 & 30 & 80 & 2 & $0,1571 \pm 0,0082$ \\
5 & 30 & 80 & 3 & $0,1694 \pm 0,0103$ \\
6 & 30 & 100 & 2 & $0,1812 \pm 0,0107$ \\
\hline
\end{tabular}

Com base nos dados apresentados na Tabela 2, verifica-se que maiores valores de coeficiente global volumétrico de transferência de massa $\left(K_{R} a\right)$ estão associados às maiores razões, velocidade de rotação dos discos e menor grau de hidratação do etanol.

\section{CONCLUSÕES}

Pode-se inferir que o processo de desterpenação do óleo essencial de bergamota modelo, utilizando como solvente o etanol hidratado é viável, sendo possível a obtenção de frações enriquecidas em compostos oxigenados. Os resultados experimentais de coeficientes de transferência de massa obtidos neste trabalho permitem avaliar a eficiência da extração em PRDC, sendo importantes para otimizar o processo de fracionamento de óleo de óleos cítricos.

\section{AGRADECIMENTOS}

Os autores agradecem à FAPESP (Fundação de Amparo à Pesquisa do Estado de São Paulo, processos $n^{\circ}$ 2010/20789-0; 2011/02476-7; 2012/15317-7; 2013/11150-3) pelo apoio financeiro.

\section{REFERENCIAS}

AGUILAR, M. S., CORTINA, J. L. Solvent extraction and liquid membranes fundamentals and applications in new materials. Boca Raton: CRC Press, 2008.

ARCE, A., MARCHIARO, A., RODRÍGUEZ, O., SOTO, A. Essential oil terpenless by extraction using organic solvents or ionic liquids. AICHE J., v.52, p.2089-2097, 2006. 


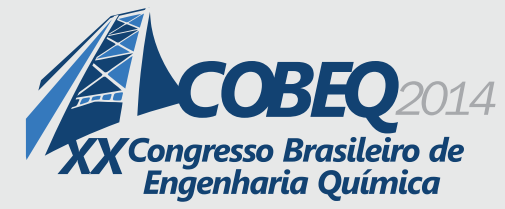

ARCE, A., MARCHIARO, A., MARTÍNEZ-AGEITOS, J. M., SOTO, A. Citrus essential oil deterpenation by liquid-liquid extraction. Can. J. Chem. Eng., v. 83, p.366-370, 2005.

ARCE, A., MARCHIARO, A., SOTO, A. Propanediols for separation of citrus oil: liquid-liquid equilibria of limonene + linalool + (1,2-propanediol or 1,3-propanediol). Fluid Phase Equilib., v.211, p.129-140, 2003.

CHIYODA, C., CAPELlini, M. C., Geremias, I. M., CARVAlHO, F. H., ARACAVA, K. K., BUENO, R. S., GONÇALVES, C. B., RODRIGUES. C. E. C. Deterpenation of bergamot essential oil using liquid-liquid. J. Chem. Eng. Data, v.56, p. 2362-2370, 2011.

GIRONI, F., MASCHIETTI, M. Phase equilibrium of the system supercritical carbon dioxide-lemon essential oil: New experimental data and thermodynamic modeling. J. Supercrit. Fluids, v.70, p.8-16, 2012.

GIRONI, F., MASCHIETTI, M. Continuous countercurrent deterpenation of lemon essential oil by means of supercritical carbon dioxide: experimental data and process modeling. Chem. Eng. Sci., v.63, p.651-661, 2008.

HAYPEK, E., SILVA, L.H.M., BATISTA, E., MARQUES, D. S., MEIRELES, M.A.A., MEIRELLES, A.J.A. Recovery of aroma compounds from orange essential oil, Braz. J. Chem.l Eng., v.17, p.705-712, 2000.

LAGO, S., RODRÍGUEZ, H., SOTO, A., ARCE, A. Alkylpyridinium alkylsulfate ionic liquids as solvents for the deterpenation of citrus essential oil. Sep. Sci. Technol., v.47, p. 292-299, 2012.

LI, H.; TAMURA, K. Ternary liquid-liquid equilibria for (water + terpene + 1-propanol or 1-butanol) systems at the temperature 298.15K. Fluid Phase Equilib., v.263, p.223-230, 2008.

PORTO, T. S., MARQUES, P. P., PORTO, C. S., MOREIRA, K. A., LIMA-FILHO, J. L., CONVERTI, A., PESSOA, A., PORTO, A. L. F. Extraction of ascorbate oxidase from cucurbita maxima by continuous process in perforated rotating disc contactor using aqueous two-phase systems. Appl. Biochem. Biotech., v. 160, p.1057-1064, 2010.

RODRIGUES, C. E. C. Desacidificação do óleo de farelo de arroz por extração líquido líquido. 221 p. (Tese de Doutorado) - Faculdade de Engenharia de Alimentos, Universidade Estadual de Campinas, Campinas, 2004.

SERAFini, L. A., SANTOS, A. C. A., TOUguinha, L. A., AgOSTINi, L. A., AgOSTini, G., DALFOVO, V. Extrações e aplicações de óleos essenciais de plantas aromáticas e medicinais. Caxias do Sul: EDUCS, 2002.

TREYBAL, R. E. Mass-transfer operations. New York: Mc Graw-Hill, 1980. 